\title{
A Young Male with Progressive Pallor
}

\author{
APARNA DAS, ${ }^{1}$ BADI UZZAMAN, ${ }^{2}$ AKM AMI NUL HOQUE, ${ }^{3}$ RATAN DAS GUPTA, ${ }^{1}$ AFROZA, ${ }^{3}$ MOHIUDDI N ${ }^{4}$, \\ ZAYED HOSSAI N ${ }^{4}$
}

\begin{abstract}
:
Dyserythropoiesis occurs in a variety of congenital and acquired haematological disorders. The term congenital dyserythropoictic anaemia was first used by Crookston et al. CDA comprises a group of rare hereditary disorders of erythropoiesis which are characterized by distinct morphological abnormalities of erythroblasts in the bone morrow. CDA is classified into three classical types (type I, II and III).Most cases of CDA are diagnosed early in life. Our patient was diagnosed at the age of 22 years with clinical presentation of anaemia, hepatomegaly and bone marrow morphology compatible with CDA type III.We report this case for rarity.
\end{abstract}

Keyword: Dyserythropoiesis, Congenital Dyserythropoictic Anaemia (CDA), Bone morrow

\section{Introduction:}

Dyserythropoiesis occurs in a variety of congenital and acquired haematological disorders. The term congenital dyserythropoictic anaemia (CDAs) was first used by Crookston et $\mathrm{al}^{1}$. These disorders are defined by their bizare red cell precursor morphology and associated serological finding. CDAs comprise a group of rare hereditary disorders of erythropoiesis which are characterized by ineffective enythropoiesis as the predominant cause of anemia and by distinct morphological abnormalities of erythroblasts in the bone morrow. Three mojor subgroups, designated type I, type II and type III and several minor subgroups have been identified, based on morphological and serological characteristics. CDA type II is frequently encountered among all CDAs ${ }^{2}$. More than 210 cases of CDAs are found. ${ }^{1-3}$

\section{Case Summary:}

A 22 years old nonsmoker, and unmarried farmer presented with progressive pallor and generalized weakness for 1 year which was associated with headache, vertigo, and palpitation .It was not associated with anorexia, loss of appetite, weight loss, any bleeding manifestations and fever. During the course of illness, he developed mild intermittent jaundice which was not associated with any prodromal symptoms, vomiting, itching, pale stool and abdominal pain \& fever. He had no significant past illness. None of his family members has similar type of illness.
Examination revealed that patient was severely anaemic, non icteric and non oedematous. His pulse was 98 beats/min, temp-normal, JVP-was not raised.No lymphadenopathy was present. He had hepatosplenomegaly which was nontender.

Investigation revealed, Hb-2.6g/dl, Hct-11\%, WBC-2.64'10\%/ L(N-64\%, L-29\%, M-5\%) \& platelet-118'10\%/L. PBF revealed severe hypochromia, with anisopoikilocytosis and microcytes and schistocytes and nucleated RBCs were seen. Bone

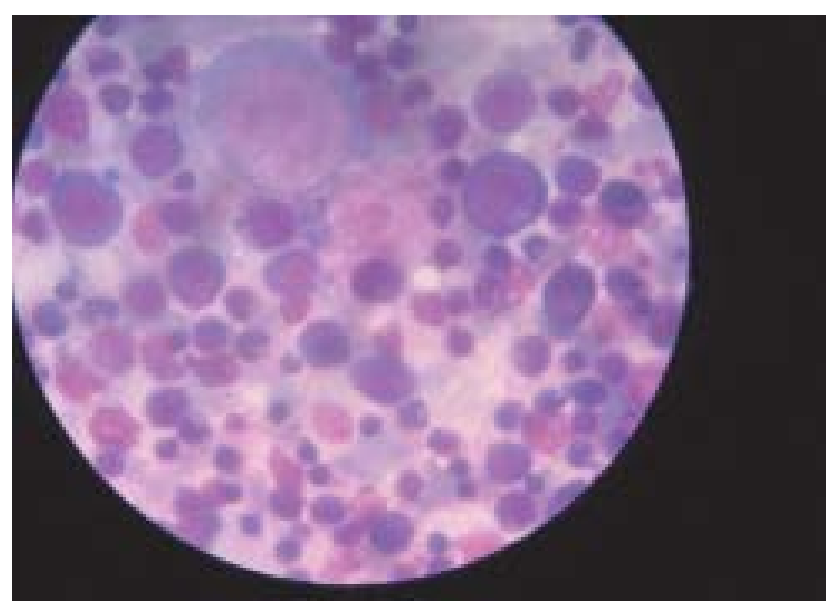

Fig.-1: Bone marrow showed hyper cellular marrow with M/E ratio decreased \& erythropoiesis was grossly hyperactive with marked dyserythropoiesis with megaloid changes, intercellular connection of erythroblasts with significant number of giant erythroblasts.

1. Asst. Professor, Department of Medicine, DMCH

2. Asst. Professor, Department of Medicine, DMCH

3. IMO, Medicine Unit-7, DMCH.

4. Associate Prof, Medicine Unit-7, DMCH

5. Associate Prof. Haematology, DMCH

6. Professor of Haematology, DMCH.

Correspondence: Dr. Aparna Das, Asst. Professor, Department of Medicine, DMC 
marrow showed hyper cellular marrow with $\mathrm{M} / \mathrm{E}$ ratio decreased \& erythropoiesis was grossly hyperactive with marked dyserythropoiesis with megaloid changes, intercellular connection of erythroblasts with significant number of giant erythroblasts. Granulopoiesis was active \& megakaryocytes were increased. Reticulocytes-01\%, bilirubin (direct- .21mg/dl, indirect- $.49 \mathrm{mg} / \mathrm{dl}$ ), serum ferritin- $8 \mathrm{ng} / \mathrm{ml}$, Hams test-negative, $\mathrm{Hb}$ electrophoresis-normal, MP-not found, ICT for kala-azar-negative, Endoscopy of upper GITnormal study.

\section{Discussion:}

CDAs are classified into 3 varieties based on distinct differences in morphology of the bone morrow erythroblasts. ${ }^{4}$ CDA type I is a well defined entity within the heterogenous group of the CDAs. ${ }^{5}$ Patients of type I usually present with Jaundice, mild to moderate anaemia and moderate splenomegaly. Ineffective erythropoiesis is the predominant mechanism of anaemia and diagnosis is based on characteristic dysplasias of the majority of polychromatic \& oxyphilic erythroblasts, with internuclear chromatin bridges being a morphological hallmark of the disorder. ${ }^{6,7}$ Patients of type II usually present with mild to severe anaemia, jaundice \& splenomegaly. Bone marrow examination shows normocytosis, bi and multinuclearity, pleuripolar mitoses with karyorrhexis. Hams test is positive, sucrose lysis test is negative and reticulocytes are low (4\%) in type II CDA. CDA type III usually present with moderate to severe anaemia and hepatosplenomegaly. Bone marrow shows macrocytoses, multinuclearity up to 12 nuclei and gigantoblasts. In 1977 David and Van Dorpe described that many cases with variable clinical features among these 3 types had subsequently occurred. ${ }^{8}$

As clinical \& laboratory findings were not distinctive, it was believed that CDAs were often underdiagnosed. ${ }^{9}$

From above case summery, this patient is consistent with CDA type III due to following reasons. First, mean age of type I \& II is 15 years but type III is 22 years with male : female $=1.9: 1$, which is consistent with our patient . Severe anaemia \& hepatosplenomegaly was present in our patient which is consistent with type III. Typical features of PBF (macrocytosis), normal unconjugated bilirubin, low reticulocytes consistent with type III. Subsequent typical bone morrow findings and negative Hams test strengthen our diagnosis. The gene responsible for CDA type III, which has been mapped to chromosome $15_{\mathrm{q}} 21-25$, is expressed not only in erythroblasts but also in B-cells and in cells of the retina ${ }^{10}$, but there was no associated B-cell lymphoma or retinal disorder in our patient. Serum ferritin was not raised in our patient.
We know that CDA type III is autosomal dominant disease. None of his parents has been suffering from this type of illness. So patient had developed CDA type III due to spontaneous mutation of the gene responsible (15q 21-25). We treated our patient with blood transfusion \& vitamins and the patient was improved.

Other typical causes of hepatosplenomegaly were excluded both by clinically and biochemically. As for example, there was absence of fever and generalized lymphadenopathy. Test for MP and ICT for kala-azar was also negative. Hb electrophorosis and endoscopy of upper GIT revealed no abnormality and HBsAg was negative.

Evaluation of anaemia in any patient requires meticulous history taking, clinical eaxamination and diagnosis is reached after adequate investigations. Our patient was provided with supportive treatment and adequate counseling.

\section{Conflict of Interest: None}

\section{References:}

1. Crookston JH, Godwin TF, Wightmann KJR, Dacie JV, Lewis SM, Patterson M, Congenital dyserythropoietic anemia. Abstr Xith Congress Int Soc Hematol, Sydney, Australia 1996

2. Wickramasinghe SN. Congenital Dyserythropoietic anaemias: Clinical features, haematological morphology and new biochemical data Blood Rev. 1998; 12:178-200

3. Congenital Dyserythropoietic Anaemia - Padebase.org. http:/ /pedbase.org/c/congenital- dyserythropoietic-anaemia/

4. Gode JS, Benz R, Fehr J, Schwarz K, Heimpel H (2006) Congenital dyserythropoietic anemia type I with bone abnormalities, mutations of the CDAN I gene and significant responsiveness to alpha interferon therapy. Ann Hematol 85:591-595 doi 10:107/s00277-006-0143-z

5. Heimpel H, wendt F. Congenital dyserythropoietic anaemia with karyorrhexis and multinuclearity of erythroblasts. Helv Med Acta. 1968; 34:103-115

6. Delaunay H, Iolascon A . The congenital dyserythropoietic anaemia: Baillieres Best Pract R Haematol.1999;12:691-705

7. Heimpel H, Schwarz K, Ebnother M, Goede J, Heydrich D, Kamp T et al (2006) Congenital dyserythropoietic anemia type I (CDA-I): molecular genetics, clinical appearance and prognosis based on long term observaton. Blood 107:334340 doi: 10.1182/blood-2005-01-0421

8. David G,and van Drope A.(1977) Abberrant congenital dyserythopoitic anaemias. In Dyserythropoisis, edited by S.M. Lewis and R.L.Verwilgehen,pp.93-100.Academic Press, London

9. Wendt F, Heimpel H (1967) Congenital dyserythropoietic anamiea bei eniem zweieiigen Zwillibgspaar. Med Kln 62:172-177

10. Wickramasinghe SN. Erythropoiesis and congenital dyserythropoietic anaemias. Br. J Hematol 1997, 98:785797 